7-1-2021

\title{
Health and ideations of married female adolescents
}

Breakthrough RESEARCH

Follow this and additional works at: https://knowledgecommons.popcouncil.org/departments_sbsr-rh

Part of the Family, Life Course, and Society Commons, and the Maternal and Child Health Commons How does access to this work benefit you? Let us know!

\section{Recommended Citation}

Breakthrough RESEARCH. 2021. "Health and ideations of married female adolescents," Programmatic Research Brief. Abuja: Population Council. 


\section{Health and Ideations of Married Female Adolescents}

This brief provides rigorous evidence-based insights to implementers and researchers of social and behavior change (SBC) programs that seek to improve knowledge, attitudes, norms, and behaviors of married female adolescents. The brief focuses on married adolescents' uptake of services and health knowledge in Kebbi, Sokoto, and Zamfara states in northwestern Nigeria using a subset of data from a household survey of married women ages 15-49 years. It is one of a series of briefs that present findings from a Breakthrough RESEARCH study that uniquely captures data on a wide range of psychosocial drivers of behavioral outcomes in the areas of family planning, malaria, and maternal, newborn, and child health, and nutrition ( $\mathrm{MNCH}+\mathrm{N})$. The results presented in this series can inform the improvement of women and children's health programming in Nigeria and help to achieve the objectives of the National Strategic Health Development Plan II (2018-2022), ${ }^{1}$ as well as support global efforts to achieve the United Nations Sustainable Development Goals. ${ }^{2}$

\section{Breakthrough RESEARCH and Breakthrough ACTION in Nigeria}

Breakthrough RESEARCH and Breakthrough ACTION are USAID's flagship SBC programs. Breakthrough ACTION in Nigeria implements SBC programming in eleven states and the Federal Capital Territory by targeting key psychosocial factors at socioecological levels (e.g., individual, community, society) in order to improve health behaviors in the areas of malaria, family planning and $\mathrm{MNCH}+\mathrm{N}$. Breakthrough RESEARCH in Nigeria conducts rigorous research to inform SBC program implementation in three of these program states (Kebbi, Sokoto and Zamfara). Findings

\section{KEY POINTS}

Married female adolescents aged 15-19 differ from their older counterparts in nearly all health behaviors, spanning such practices as the use of antenatal care (ANC), postpartum contraception, breastfeeding, childhood vaccinations, and treatment of childhood illnesses.

More than half of female adolescents $(54.2 \%)$ are married to someone ten or more years their age, which likely has implications for agency, spousal communication, joint decision-making, and other cross-cutting drivers of health behaviors.

Ideational factors-particularly knowledge of the benefits of health services and self-efficacy to act-appear to explain much of these differences. Health knowledge among adolescents lags behind that of older women. For nearly all behaviors (e.g., contraceptive use, ANC, facility delivery, breastfeeding, treatment for child illnesses), married female adolescents express less confidence in their abilities both to convince their husband about a course of action and to engage in that action, potentially contributing to lower service utilization and lesser engagement in healthy behaviors.

Other factors-such as education, wealth, and age differentials with husbands-do not differ across age groups and hence are unlikely to explain differences in behaviors.

Perhaps surprisingly, decision-making autonomy does not differ significantly between adolescents and older women. Husbands appear to be as important for health decisions amongst adolescents as among older women. The majority of adolescents, like their older counterparts, support communication with their spouses about health topics. 
presented here are from a Breakthrough RESEARCH baseline study that are used for informing SBC program adaption and scale-up in Nigeria.

\section{Setting the context}

Adolescent girls in northern Nigeria typically face limited educational opportunities, and frequently experience early marriage and early childbirth, all of which are associated with greater health risks, including pregnancy complications that contribute to both neonatal and maternal morbidity and mortality. These factors are more pronounced in the northwest of Nigeria than elsewhere in the country. The median ages at first marriage (15.9 years) and first birth (18.1 years) are lower in the northwest than in any other region of Nigeria. ${ }^{3}$ According to the 2018 Nigeria Demographic Health Survey (NDHS), nearly one-third (31.0\%) of 15-year-old girls in the northwest are married, and, by the age of 18, almost one-third have a child.

The 2018 NDHS also shows that women's empowerment and decision-making autonomy are lower in the northwest than in any other region. ${ }^{3}$ The majority of adolescent marriages are arranged by families, often against a girl's wishes and frequently with a considerably older man. ${ }^{3}$ Only $36.9 \%$ of women in the 2018 NDHS reported that they could say no to their husband if they do not want to have sexual intercourse.

Compounding these issues, many adolescent girls have limited knowledge of basic sexual and reproductive health, as well as limited awareness of key maternal and child health services. ${ }^{4}$ A 2015 study by the World Bank found that only $6.6 \%$ of 15 - to 19 -year-old Nigerian females were able to identify when a female is most likely to get pregnant during her ovulatory cycle. ${ }^{5}$ The 2018 NDHS indicated that more than four out of ten 15- to 19-year-olds in the northwest were unable to identify a single modern contraceptive method. ${ }^{3}$ Part of this knowledge deficit may be due to reduced access to information sources among adolescents and young mothers. Studies have revealed that married adolescents have less access to media than their unmarried counterparts and face greater barriers in accessing health services. This leaves adolescents in a position of being ill-prepared for pregnancy and childbirth, events which can be "confusing and traumatic."4

\section{Objectives}

- Estimate differences in key health behaviors for married female adolescents relative to their older counterparts, and specifically in the areas of postpartum family planning, ANC, delivery care,

breastfeeding, care-seeking for childhood illnesses, and childhood immunizations.

- Explore differences in ideational factors that may help to explain differences in health behaviors between adolescents and older women.

\section{Study methods}

Results are based on the behavioral sentinel surveillance (BSS) baseline survey conducted between September and October 2019 in Breakthrough ACTION program areas in Kebbi, Sokoto, and Zamfara States of northwestern Nigeria. The baseline survey included 3,000 married postpartum women aged 15-49 years, including 353 adolescents aged 15-19 years. Figure 1 summarizes the survey methods.

The analysis presents weighted descriptive statistics for married female adolescents aged 15-19 years relative to married women aged $20-29$ years and $30-49$ years. Both the bivariate and multivariate analyses are linked with ideational measures related to health behaviors and health services, including: (1) knowledge of the benefits, (2) myths, (3) beliefs, (4) values and injunctive norms, (5) descriptive norms, (6) self=efficacy, (7) social influences,

\section{FIGURE 1 BSS BASELINE SURVEY METHODS}

Study population

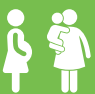

Pregnant women and women with a child under 2 years old living within Breakthrough ACTION program areas in Kebbi, Sokoto and Zamfara states (not representative at state level)

Study

design

res

Cross-sectional

Sample

size

t?

\#

pregnant women

3,043 women with a child under 2 years

Sampling

method

마요

$\because$

108 wards across 3 states

Census of pregnant women

Random selection of women with a child under 2 years

Data Mixed-effects logistic regression models were analysis used to derive predicted probabilities of nutrition outcomes, controlling for behavioral and ideational outcomes and sociodemographic characteristics, including household wealth, education (woman and spouse), and employment (woman and spouse). 
and (8) interpersonal communication with a partner and health workers. To illustrate the influence of ideational factors for adolescents compared to older women, a multivariate logistic regression model was used to quantify the associations between the practice of immediate breastfeeding after birth and sociodemographic and ideational measures. Post-estimation analysis calculates average marginal effects, or the change in the probability of immediately breastfeeding when a predictor variable, e.g., an ideational factor, increases by one unit.

\section{Key results}

\section{Differences in health behaviors}

Female adolescents are less likely to engage in nearly all positive $\mathrm{MNCH}+\mathrm{N}$ behaviors explored (Figure 2). Adolescents are less likely (14\%) to make at least four ANC visits as women aged 20-29 (23\%) and women aged 30-49 (29\%) and to receive intermittent preventive treatment during pregnancy (IPTp) (15-19: 11\%, 20-29: 19\%, 30-49: $23 \%)$, thereby placing adolescents at greater risk of adverse pregnancy outcomes. Moreover, even among women who made at least one ANC visit, adolescents are less likely to discuss with health providers key topics such as vaginal bleeding, baby's movements, due dates, danger signs during pregnancy, delivery plans, the presence of family members during delivery, transport, funds for delivery, and finding a skilled birth attendant, further increasing pregnancy and delivery risks. In the postnatal period, adolescents are much less likely (27\%) to begin breastfeeding within one hour of birth relative to mothers aged 20 to 29 years (43\%) and mothers aged 30 to 49 years (45\%). Similarly, for common childhood illnesses, adolescents are less likely to provide oral rehydration salts (ORS) and zinc for children with diarrhea (15-19: 18\%, 20-29: 19\%, 30-49: $26 \%$ ) and less likely to seek formal medical care for children with symptoms of pneumonia (15-19: 22\%, 20-29: 28\%, 30-49: 40\%). For pediatric fever, the differences across age groups in the percentage who sought care from formal medical sources were also large (15-19: 28\%, 20-29: 38\%, 30-49: 46\%). Only 8\% of married adolescents aged 15-19 years were currently using modern contraception to space or limit pregnancies, relative to $13 \%$ of $20-29$ year olds and $15 \%$ of $30-49$ year olds.

The remainder of this brief examines differences in both sociodemographic and ideational factors that may influence observed differences in health behaviors between married adolescent and older mothers.

\section{FIGURE 2 KEY MNCH+N BEHAVIORS BY MATERNAL AGE}

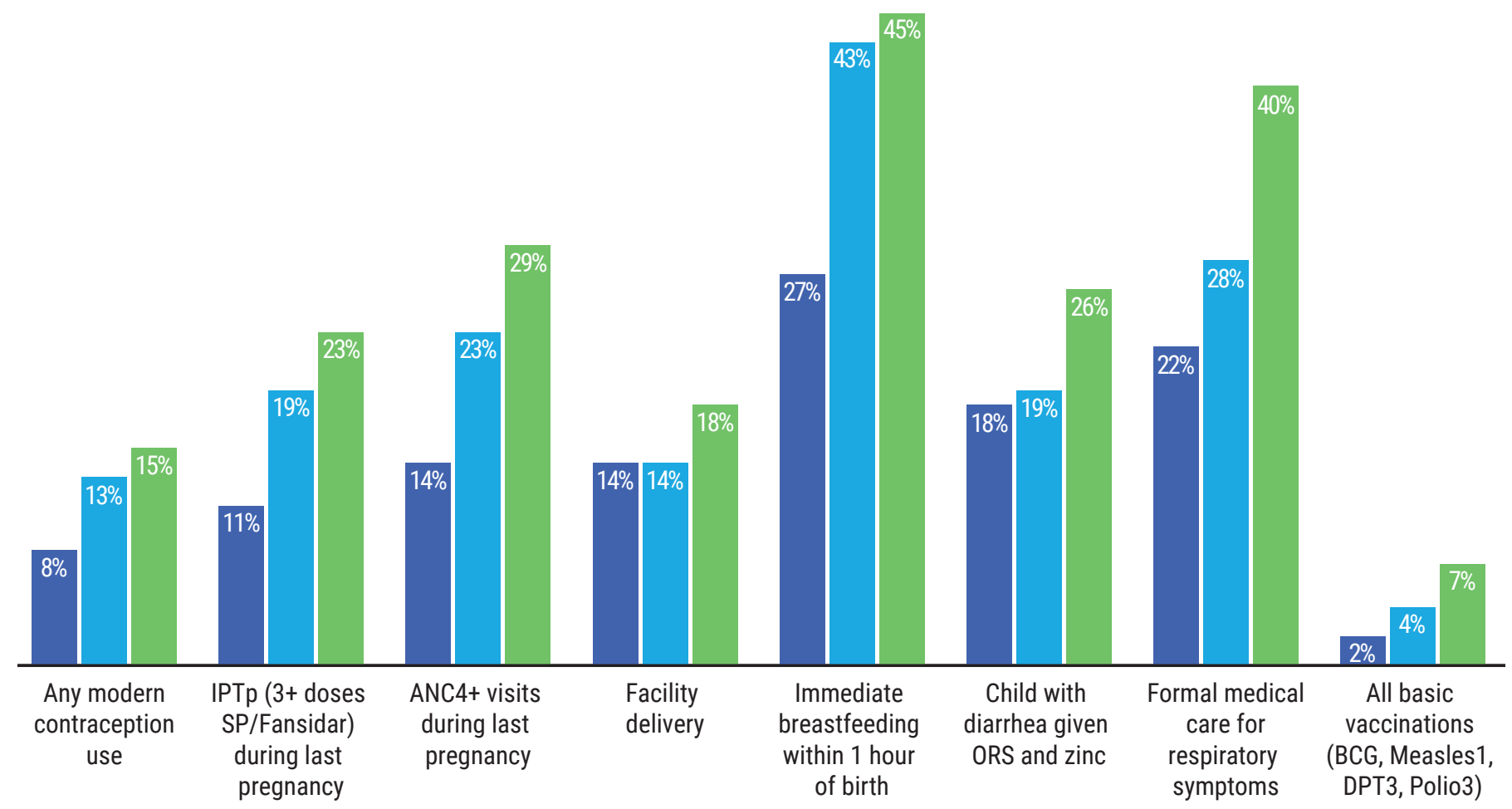


Sociodemographics: Adolescent mothers appear no different than older mothers in terms of their sociodemographic characteristics, including levels of education, wealth, and husband's education. As such, sociodemographic variables are unlikely to explain differences in health behaviors across age groups. Differences in empowerment for household decision-making also appear unlikely to explain differences in outcomes as older women do not appear to have any greater autonomy with respect to decisions about how the woman's money will be used, how the husband's money will be used, major household purchases, schooling, and working outside the home.

Age difference with husbands: Relative to older women, adolescents are less likely to have a husband that is their age-peer, namely a person who is within four years of her age. More than half of adolescents (54\%) are married to someone 10 or more years their age, which likely has implications for agency, spousal communication, joint decision-making, and other cross-cutting drivers of health behaviors.

Knowledge: Health knowledge among adolescents lags that of older women with more children, potentially contributing to lower service utilization and less engagement in healthy behaviors. Adolescents are less likely to know all methods of contraception than older women and have lower contraceptive knowledge overall. For example, adolescents are less likely to spontaneously mention awareness of injectable methods (15-19: 43\%, 20-29: 57\%, 30-49: 58\%), implants (15-19: 36\%, 20-29: 49\%, 30-49: $49 \%$ ), and oral contraceptives (15-19: 28\%, 20-29: 38\%, 30-49: 46\%). Adolescents are less likely to know of health benefits to the mother or the child from contraceptive use or ANC. For example, only $10 \%$ of adolescents cite reduction in risk of pregnancy complications as a benefit of ANC relative to $17 \%$ of women aged $20-29$ and $17 \%$ of women 30-49.

Adolescents may also have less experience with other family health issues, which may contribute to perceptions of their need for health services and ultimately lower service utilization than older women. Adolescents are less likely to know that women over 35 years are at higher risk of pregnancy complications (15-19: 31\%, 20-29: 41\%, 30-49: $41 \%)$, breastmilk contains essential nutrients for the first six months of life (15-19: 75\%, 20-29: 87\%, 30-49: 89\%), a blood test is the only way to know if a person has malaria (15-19: 31\%, 20-29: 41\%, 30-49: 41\%), antibiotics are the most effective treatment for pneumonia (15-19: 47\%, 20-29: 59\%, 30-49: 64\%), or how to prepare ORS (15-19: 59\%, 20-29: 69\%, 30-49: 73\%).
Autonomy and decision-making: Decision-making autonomy does not differ significantly between adolescents and older women. Husbands appear to be as important for health decisions amongst adolescents as among older women. Roughly $32 \%$ of adolescents report that their husband influences decisions about the use of modern contraception relative to $29 \%$ of $20-29$ yearolds and $32 \%$ of 30- to 49-year-olds. Similarly, the decision to deliver in a health facility is almost equally influenced by husbands for $60 \%$ of adolescents relative to $57 \%$ of 20 - to 29 -year-olds and $58 \%$ of 30 - to 49 -year-olds. For other services, including childhood immunizations and care-seeking for sick children, there are also no statistically significant differences in husbands' influence across age groups. Adolescents are more likely to report being influenced by mothers and mothers-in-law relative to older women, although such influence is relatively uncommon among all women.

In general, husband's opposition as a reason for non-use of services is no more or less common for adolescents than for older women, with the exception of ever breastfeeding. In that case, $53 \%$ of adolescents who did not breastfeed cited partner's opposition, relative to only $29 \%$ of 20 - to 29 -year-old mothers and $18 \%$ of 30 - to 49 -year-old mothers.

Self-efficacy: For nearly all behaviors, adolescents express less confidence in their abilities both to convince their husband about a course of action and to engage in that action. Relative to older women, they are less likely to be confident that they can use modern contraception if a partner disagrees (15-19: 27\%, 20-29: 38\%, 30-49: 41\%), get to a health facility for ANC (15-19: 60\%, 20-29: 69\%, 30-49: 69\%), get to a health facility for delivery (15-19: 37\%, 20-29: 44\%, 30-49: 48\%), and exclusively breastfeed (15-19: 50\%, 20-29: 56\%, 30-49: 58\%. Relative to women aged $20-29$ years and $30-49$ years, they are less likely to be confident that they can start a conversation with a husband about attending ANC (15-19: 66\%, 20-29: 73\%, 30-49: 73\%), about facility delivery (15-19: 41, 20-29: 51\%, 30-49: 53\%), and about breastfeeding (15-19: 61\%, 20-29: 71\%, 30-49: 70\%). Adolescents are confident, however, that they can convince their husbands to seek care for cough with rapid breathing (15-19: 85\%, 20-29: 90\%, 30-49: 90\%) and to seek care for a sick child (15-19: 88\%, 20-29: 92\%, 30-49: 93\%).

Attitudes: In some areas, adolescents appear more reticent about health services than older women. For example, they are less likely to approve of modern contraception and less likely to agree that couples who use family planning have a better quality of life. They are less likely to believe that women attending four or more ANC visits 
have safer pregnancies, or that the illnesses prevented by vaccines are serious, or that people in their community trust immunization services. On the other hand, in some areas, adolescents are not remarkably different than older women. They are no more or less likely to believe that pregnant women only need ANC when sick or that only first time pregnant women need ANC. They are no more likely to believe that breastmilk after birth is bad nor to believe that contraceptives can cause cancer.

Interpersonal communication: The majority of adolescents, like their older counterparts, support communication with their spouses about health topics. For example, approximately 9 in 10 women of all ages believe that it is important for a woman to discuss her pregnancy with her husband, and approximately $95 \%$ of both adolescents and older women believe that it is important for couples to discuss and decide together about child health. Nonetheless, adolescents are only about two-thirds as likely as women aged 20-29 years to have ever talked with their husband about the number of children to have or about using modern contraception, indicating a potential missed opportunity to transform desires for spousal communication into actual conversations about important health issues.

\section{Multivariate analyses}

The small number of observations ( $n=353$ ) for married adolescent women aged 15-49 years coupled with overall low prevalence of undertaking priority health behaviors potentially contributed to findings of non-significant associations between ideations and behaviors for this age group across most malaria, family planning, and $\mathrm{MNCH}+\mathrm{N}$ behaviors.

For immediate breastfeeding after birth, where there was a large difference in behaviors for adolescent and older women, regression analyses indicated how ideational factors could influence differences in health behaviors between adolescents and older women. Figure 3 presents the likelihood that a married adolescent woman will immediately breastfeed based on her level of knowledge of breastfeeding practices and of the benefits of breastfeeding to herself and baby. Each of these factors is statistically associated with the likelihood of breastfeeding and importantly each of these factors differ markedly between adolescents and older women.

For example, nearly $90 \%$ of mothers $30-49$ years knew that breastmilk contains all of the essential nutrients for the health of the infant for the first six months of life but

FIGURE 3 LIKELIHOOD OF IMMEDIATE BREASTFEEDING AMONG MARRIED ADOLESCENT MOTHERS BY IDEATIONAL FACTOR

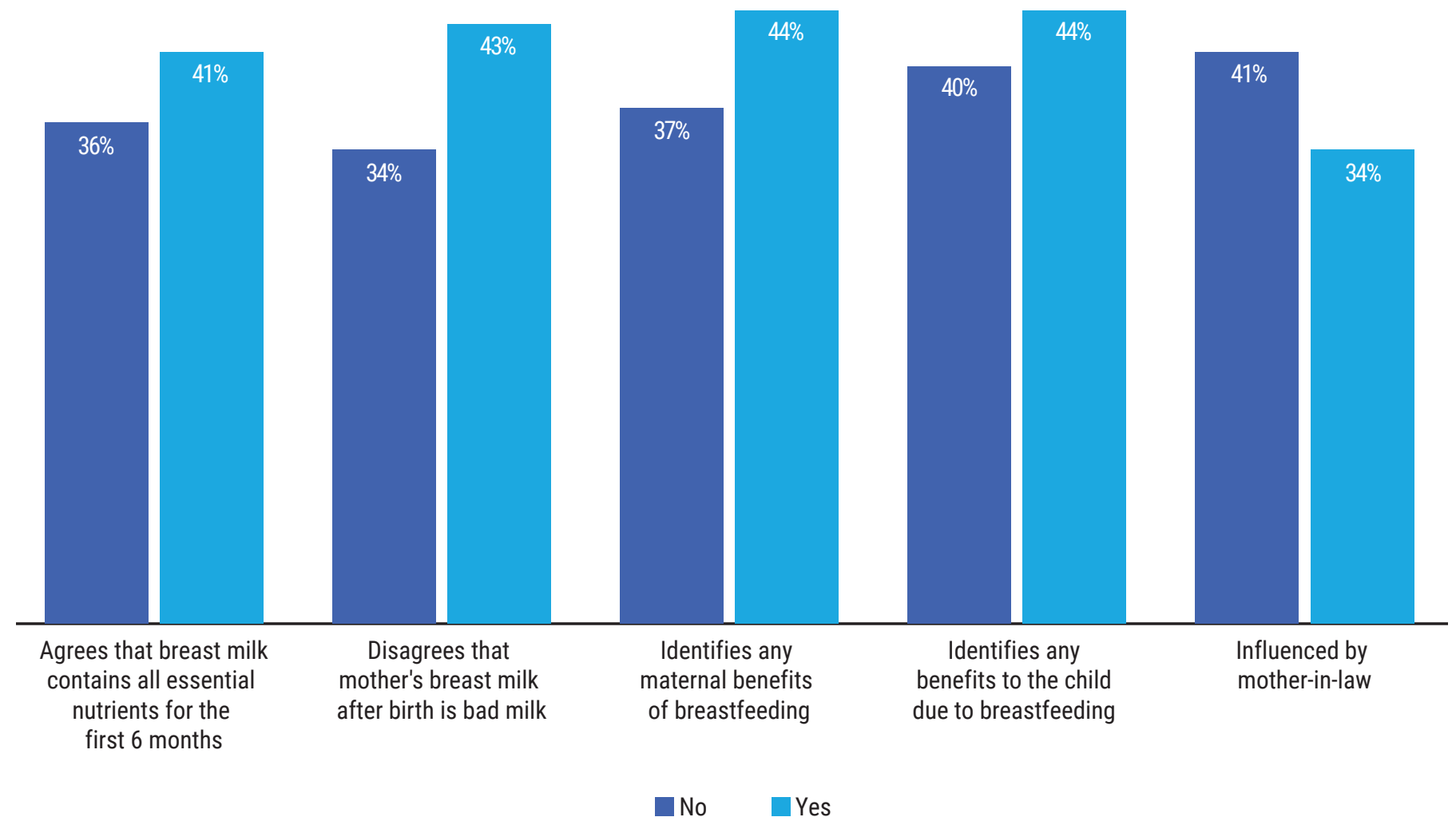


only $75 \%$ of adolescents had such awareness. This difference is important because mothers who knew this fact were 4.4 percentage points more likely to immediately breastfeed ( $41 \%$ versus $36 \%$ ). Similarly, $48 \%$ of adolescents were able to identify any benefit to the mother of breastfeeding relative to $54 \%$ of mothers aged $30-49$ years. Such knowledge appears critical because women who can identify benefits of breastfeeding to herself are 6.8 percentage points more likely to immediately breastfeed their newborn within 24 hours of childbirth (44\% versus $37 \%$ ).

Knowledge is not the only crucial factor. Autonomy is also statistically related to immediate breastfeeding practices among married adolescent mothers. For example, adolescents whose breastfeeding decisions are influenced by their mother-in-law are 6.2 percentage points less likely to immediately breastfeed their newborn within 24 hours of birth (41\% versus $34 \%$ ). Notably, nearly $10 \%$ of adolescents are influenced in breastfeeding by their mother-in-law versus only $6 \%$ of women aged $30-49$ years.

\section{Conclusions}

The conclusions that can be drawn from these analyses about married female adolescents' health behaviors and ideations are limited due to the relatively small sample size of this age group in our baseline survey. Largely for this reason, we found no significant associations between ideations and behaviors for this age group across most malaria, family planning, and $\mathrm{MNCH}+\mathrm{N}$ behaviors. This limits our recommendations about the most effective SBC interventions or messaging across different health areas that could be targeted specifically to young mothers to improve behavioral outcomes.

Nevertheless, descriptive statistics for married female adolescents compared to older women provide important insights into differences in health behaviors and some of the ideations that may potentially drive these differences. This is also reinforced by regression analysis results for immediate breastfeeding practices. While there were limited socioeconomic differences across age groups, some ideational factors could play an important role, such as lower health knowledge across many areas (including breastfeeding) and worse attitudes about health services among married adolescent women compared to older women. Lower knowledge and less positive attitudes about health services could suggest important areas for targeting youth-friendly SBC programming going forward. Adolescent mothers also had lower self-efficacy and confidence in performing these behaviors or accessing health services (e.g., contraceptive use, ANC, facility delivery, breastfeeding, treatment for child illnesses), which could be due to their relative inexperience with family health behaviors, such as child care-seeking and treatment for common illnesses.

In addition, while all women cited spouses as a main influence in health decision-making, adolescent women were more likely to cite mothers and mothers-in-law as influencing decisions, which was also a significant result for immediate breastfeeding. This suggests another potential communication channel for specifically targeting SBC programs such that mothers and mothers-in-law are also included in youth-friendly SBC programming given their influence on young mothers' behaviors. This merits further exploration through qualitative studies and future research. Finally, while all women had a stated desire for improved spousal communication for family planning, married adolescent women were less likely to engage in actual conversations with their spouses about family planning, which could potentially also be related to spousal age differences, lack of self-confidence or self-efficacy, or limited knowledge or worse attitudes about health services.

\section{Appendix}

\section{Outcomes and behaviors}

The proposed analysis will focus on key behaviors targeted by the social and behavior change program of Breakthrough ACTION, including:

- Current modern contraceptive use

- Antenatal care (4+ visits)

- Intermittent Preventive Treatment in Pregnancy (IPTp) for malaria

- Facility delivery

- Immediate breastfeeding after birth

- Exclusive breastfeeding for 6 months after birth

- Formal treatment for fever, diarrhea and acute respiratory infections

- Treatment with ORS and zinc for childhood episodes of diarrhea

- Full immunization for children

\section{Predictor variables}

\section{Sociodemographic variables}

- Maternal education

- Maternal age

- Household wealth

- Spousal education

- Spousal age difference 
Ideational variables

\section{Cognitive}

- Knowledge of benefits of health behaviors and health services

- Myths related to health behaviors and health services (e.g., that "colostrum is bad milk")

- Beliefs regarding health behaviors and health services

- Values and injunctive norms (e.g., approval)

- Descriptive norms

\section{Emotional}

- Self-efficacy to engage in health behaviors

\section{Social}

- Social influence on using health services

- Interpersonal communication with partner and health workers 


\section{References}

1. Federal Government of Nigeria. 2019. National Strategic Health Development Plan II (NSHD) 2018-2022. Abuja: Federal Government of Nigeria.

2. United Nations General Assembly. 2015. Transforming our work: the 2030 agenda for sustainable development. New York: United Nations.

3. National Population Commission (NPC) [Nigeria] and ICF. 2019. Nigeria Demographic and Health Survey 2018. Abuja, Nigeria, and Rockville, Maryland, USA: NPC and ICF.

4. Erulkar, A. S. and M. Bello. 2007. "The experience of married adolescent girls in northern Nigeria." New York: Population Council.

5. Cortez, R. et al. 2015. "Adolescent sexual and reproductive health in Nigeria," HNPGP Knowledge Brief. Washington, D.C.: World Bank.

\section{Acknowledgments}

This programmatic research brief describes work led by Tulane University under Breakthrough RESEARCH. This brief and the work it describes is possible through the work and support of the Center for Research, Evaluation Resources and Development (CRERD), Breakthrough ACTION in Nigeria, Population Reference Bureau, and Population Council in Washington, DC.

\section{Suggested citation}

Breakthrough RESEARCH. 2021. "Health and ideations of married female adolescents - Insights for improving malaria, family planning, and maternal and child health outcomes in northwestern Nigeria through social and behavior change programming," Programmatic Research Brief. Abuja, Nigeria: Population Council.

(C) 2021 The Population Council. All rights reserved.

\section{Email}

\section{BreakthroughResearch@popcouncil.org}

\section{Breakthrough RESEARCH | Population Council}

Plot 839 Idris Ibrahim Crescent | Jabi, Abuja, Nigeria +2348067787750

4301 Connecticut Ave., NW, Suite 280 | Washington, DC 20008 +12022379400 | breakthroughactionandresearch.org
Breakthrough RESEARCH is made possible by the generous support of the American people through the United States Agency for International Development (USAID) under the terms of cooperative agreement no. AIDOAA-A-17-00018. The contents of this document are the sole responsibility of Breakthrough RESEARCH and Population Council and do not necessarily reflect the views of USAID or the United States Government.
POPULATION COUNCIL

Ideas. Evidence. Impact.

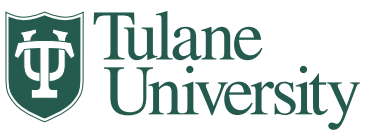

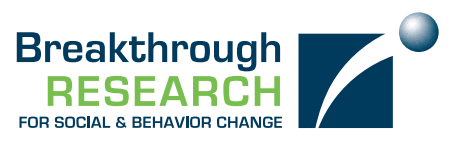

Our project turns evidence into action by providing thought provoking guidance to improve SBC policy and programming, with the goal of improving the cost-effectiveness of USAID's health and development strategies. Breakthrough RESEARCH catalyzes SBC by conducting state-of-the-art research and evaluation and promoting evidence-based solutions to improve health and development programs around the world. Breakthrough RESEARCH is a consortium led by the Population Council in partnership with Avenir Health, ideas42, Institute for Reproductive Health at Georgetown University, Population Reference Bureau, and Tulane University. 